\title{
Recurrent Right Upper Quadrant Pain Masquerading an Underlying Colon Adenocarcinoma-Induced Intussusception
}

\author{
Zeena Ayad Qiryaqoz $^{a} \quad$ Urvashi Katiyar $^{\mathrm{b}}$ Saad Shebrain ${ }^{\mathrm{b}}$ \\ aWestern Michigan University Homer Stryker, School of Medicine, Kalamazoo, MI, USA; \\ bBronson Methodist Hospital, Kalamazoo, MI, USA
}

\section{Keywords}

Adenocarcinoma $\cdot$ Case report · Gastroenterology $\cdot$ Intussusception

\begin{abstract}
Intussusception in adults is a challenging diagnosis that often requires a high degree of suspicion. In adults presenting with symptoms, almost $90 \%$ have underlying neoplasms. Most frequently, the presentation will include nonspecific abdominal pain, vomiting, and mucoid hematochezia. In this case, we present a 39-year-old female with a rare presentation of chronic, recurrent right upper quadrant abdominal pain over a 5-month interval. The misleading presentation with which the patient presented led to a delay in diagnosis and treatment of colon malignancy and serves to advocate for intussusception as a differential for adult patients presenting with obstructive symptoms of unknown origin and recurrent abdominal pain. Such cases should persuade physicians to plan prompt surgical intervention as to not delay optimal diagnostic and therapeutic outcomes.
\end{abstract}

\section{Introduction}

Intussusception is a potentially life-threatening condition that commonly presents with intermittent abdominal pain and cramping and may lead to ischemia of the small bowels. The clinical presentation classically involves abdominal pain and/or vomiting, as well as mucoid hematochezia (i.e., red currant jelly stool). Intussusception is a common cause of pediatric intestinal obstruction [1]. Adult intussusceptions, however, are rare, accounting for $<5 \%$ of cases of all intussusception and 1-5\% of all bowel obstruction [2]. Unlike the pediatric population in which the cause is most frequently idiopathic, adults have an underlying pathologic lesion in $80-90 \%$ of cases, with a malignant lead point present in $70 \%$ of adult colonic intussusception 
Fig. 1. Abdominal/pelvic CT showing colocolonic intussusception at the hepatic flexure (yellow arrow).

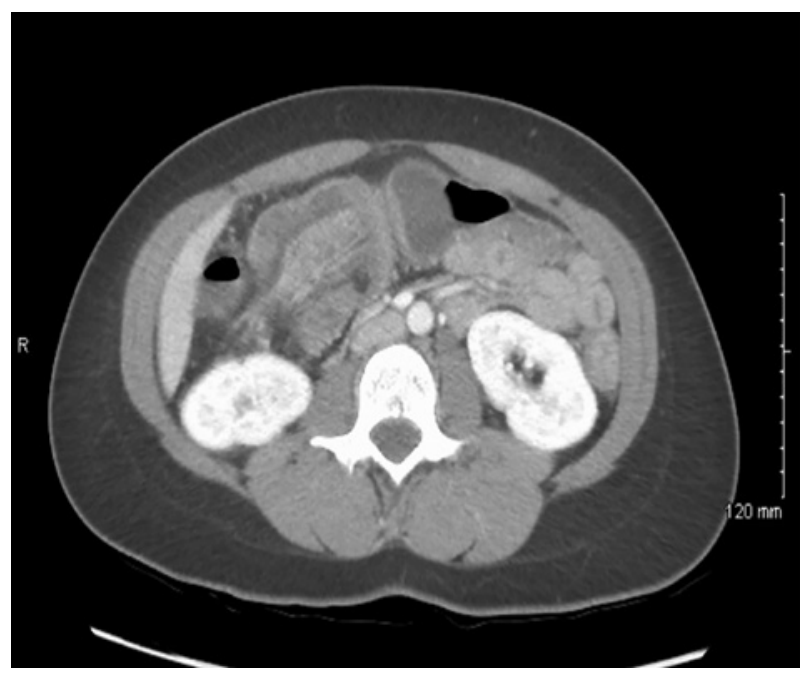

cases [3]. Adenocarcinoma accounts for up to $30 \%$ of small bowel intussusception [3]. Oncologic resection remains the optimal therapeutic option for such cases. We report an atypical presentation of a 39-year-old female with intussusception due to an underlying ascending colon adenocarcinoma.

\section{Case Report/Case Presentation}

A 39-year-old African American female presented with chronic, recurrent right upper quadrant (RUQ) pain over a 5-month interval. She was otherwise healthy. Upon out-patient workup for biliary diseases, including RUQ ultrasound, there was no evidence of gallstones. Subsequently, a hepatobiliary iminodiacetic acid scan with parenteral injection of cholecystokinin (CCK) was performed to assess the functional status of the gallbladder and demonstrated the maximum gallbladder ejection fraction to be 50\% (a normal gallbladder ejection fraction is $35 \%$ or greater within $30 \mathrm{~min}$ after CCK injection). The patient's symptoms persisted and worsened in severity and frequency, forcing the patient to seek urgent medical care in the Emergency Department (ED). Two days prior to visiting the ED, she reported the sensation of food getting "stuck" in her stomach, lasting for several minutes, relieved with defecation. She denied fevers, hematochezia, hematemesis, or changes in bowel/urinary habits. Initial physical examination demonstrated normal vitals aside from hypertension (150/97 mm Hg). Cardiopulmonary exam was unremarkable. Abdominal exam revealed a soft, minimally tender lower abdomen, more pronounced at the right lower quadrant with no guarding, palpable masses, or organomegaly. Laboratory tests included complete blood count and basic metabolic panel: all values normal except WBC 4,000/mL (L). Liver function tests were all normal except alkaline phosphatase $41 \mathrm{U} / \mathrm{L}$ (L). A CT scan of the abdomen/pelvis demonstrated an intussusception at the hepatic flexure of the colon and the cecum mildly distended with fluid and gas, implying the intussusception may be obstructing (shown in Fig. 1). Further evaluation with a Gastrografin contrast enema illustrated a reduced intussusception but revealed an irregular narrowing of the ascending colon, raising suspicion for a mass (shown in Fig. 2). The patient was admitted to the hospital, started on parenteral fluids, and a gentle bowel preparation was administered using low volumes of polyethylene glycol (Golytely ${ }^{\circledR}$ ). Colonoscopy was then performed and revealed a friable, fungating mass in the ascending colon. Subsequent biopsy confirmed the presence of infiltrating colorectal adenocarcinoma with features of low-grade neoplasm. 
Fig. 2. Barium enema showing reduced intussusception with irregular narrowing of the ascending colon (yellow arrow).

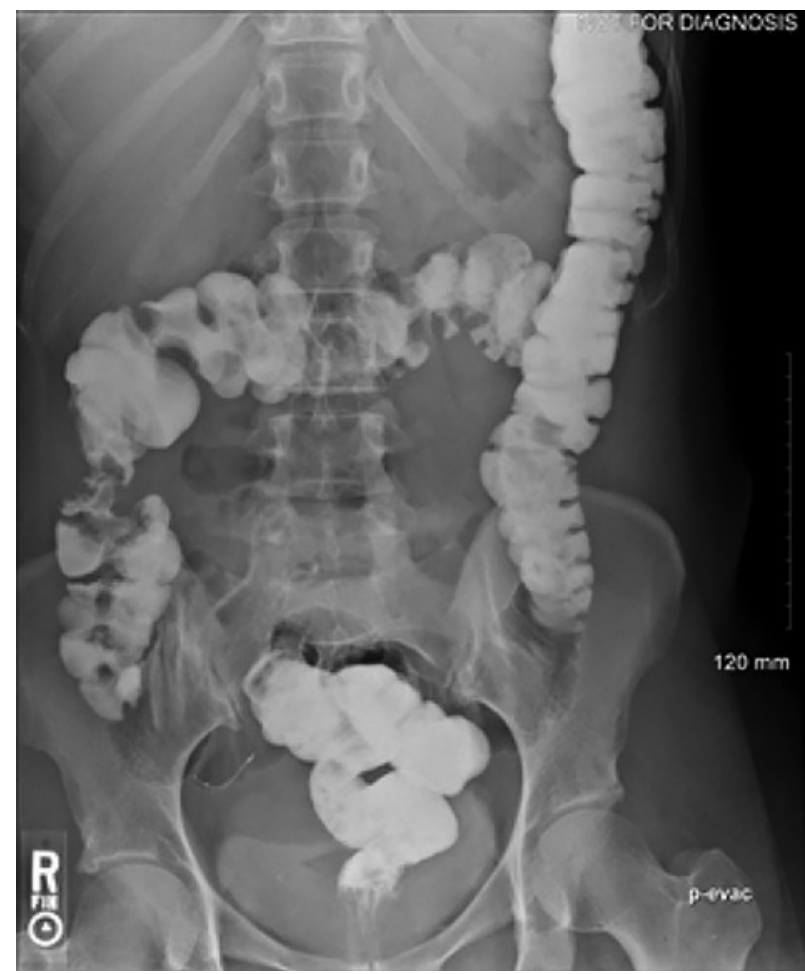

Fig. 3. The terminal ileum measured $6.5 \times 1.9$ $\mathrm{cm}$. The cecum and colon are $22 \mathrm{~cm}$ long and range in diameter from $3.5 \mathrm{~cm}$ at the distal end to $5.9 \mathrm{~cm}$ near the midpoint.

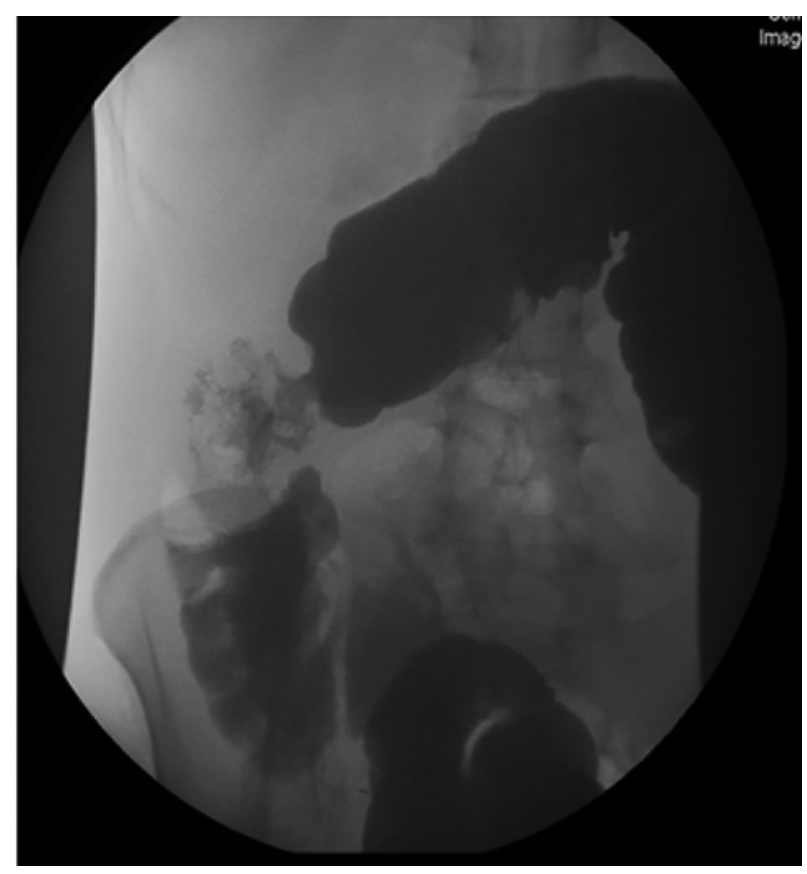

Gross surgical pathology demonstrated terminal ileum, cecum with attached appendix, and proximal portion of the colon. The terminal ileum measured $6.5 \times 1.9 \mathrm{~cm}$. The cecum and colon were $22 \mathrm{~cm}$ long and ranged in diameter from $3.5 \mathrm{~cm}$ distally to $5.9 \mathrm{~cm}$ near the midpoint (shown in Fig. 3). Final pathology demonstrated ascending colon adenocarcinoma with angiolymphatic invasion and one out of 28 lymph nodes with metastatic disease (T3, N1, M0). Microsatellite instability screen was negative. The tumor exhibited normal nuclear expression 
of mismatch repair proteins MLH-1, MSH-2, MSH-6, and PMS-2, indicating a microsatellite stable tumor. The patient subsequently underwent surveillance colonoscopies with no recurrence at 7 years.

\section{Discussion/Conclusion}

Intussusception is a pathology in which a fragment of the bowel invaginates into a distal neighboring segment. This condition typically involves an insult in the bowel wall that acts as a lead point that may lead to changes in the normal tissue leading to intussusception [4]. The lead point is pulled forward by normal peristalsis, telescoping or prolapsing the affected segment of the bowel (intussusceptum) into another segment of the bowel (intussuscipiens) [5]. Most commonly this then occurs in locations in the bowel that are progressively increased in size, such as the ileocolonic junction. In adults, $52 \%$ of surgically diagnosed intussusceptions were found in the small intestine and $38 \%$ in the large intestine [6].

When intussusception occurs without a lead point, it is referred to as primary and is more likely to occur in the small intestines. In contrast, a secondary intussusception occurs when a lead point is identifiable. Secondary intussusception is caused by organic lesions, such as inflammatory bowel disease, postoperative adhesions, Meckel's diverticulum, benign and malignant lesions, metastatic neoplasms, or even iatrogenic lead points, due to the presence of intestinal tubes, jejunostomy feeding tubes, or after gastric surgery [5].

Interestingly, adults account for $<5 \%$ of all cases of intussusception. In adults, intussusception has an identifiable etiology in $90 \%$ of cases [6]. In the small intestine, benign lesions represent most lead points, and only $30 \%$ are malignant lesions, most commonly metastasis. In comparison, $>65 \%$ of cases of intussusception that occur in the colon have a malignant etiology, most commonly adenocarcinoma [7].

We reported a rare presentation of colonic intussusception that was induced by an underlying adenocarcinoma in an adult patient. These patients often present with nonspecific abdominal pain and other symptoms of bowel obstructions. The classic triad of RUQ pain, currant jelly stools, and bilious vomiting present in children is possible but rarely seen in adults. Patients most often present with symptoms consistent with intermittent partial bowel obstruction such as nausea, vomiting, melena, weight loss, fever, and constipation with intermittent cramping pain [8].

Biliary colic (symptomatic cholethiasis) occurs when a gallbladder stone blocks the cystic duct upon gallbladder contraction stimulated by CCK, an enzyme secreted by the duodenum in response to fat and protein. CCK causes the gallbladder to contract, pushing any gallstones to the neck of the gallbladder and causing a colicky pain that lasts very briefly (15 min to an hour) and is associated with the consumption of fatty meals. The gastrocolic reflex involves the increase in motility and peristalsis stimulated by distension of the stomach from food. It has been implicated in the pathogenesis of inflammatory bowel syndrome, as overstimulation of this reflex can lead to postprandial abdominal pain and diarrhea. Both IBS and biliary colic are common diagnoses for patients presenting with vague complaints of chronic, intermittent abdominal pain, and a physician must be hypervigilant to rule out an intussusception prior to arriving at these more common diagnoses.

If suspected, plain abdominal films can show signs of bowel obstruction such as narrowing and air fluid levels. However, a CT is recommended to demonstrate the pathognomonic "target sign" of intussusception as well as help identify malignant lead points. In adults, CT scans can also help delineate intussusception requiring surgical intervention from those that are self-limiting. Lvoff et al. [9] found that in adults, a small bowel intussusception that is shorter than $3.5 \mathrm{~cm}$ is likely to be self-limiting.

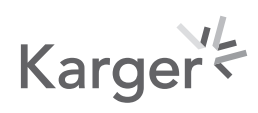


Children can often be managed nonoperatively for symptomatic intussusception with air contrast enemas, as their most common etiology is idiopathic. Due to the rate of malignant etiology in adults, exploratory laparotomy for reduction or resection of lead point mass or area of ischemia is recommended. The only instance in which preoperative reduction in adults is recommended is if the lead point is previously known to be benign or the tissue is known to be viable or where resection may result in short gut syndrome. No intervention is required on asymptomatic patients with incidental findings of nonobstructing intussusception on CT [10].

Here, we presented a 39-year-old patient with nonspecific complaints that led to a significant delay in diagnosis. Recurrent abdominal pain is an extremely common and challenging chief complaint in the ED. The diagnosis can often be delayed due to the vague and intermittent nature of the complaint. We advocate for intussusception as a differential for adult patients presenting with obstructive symptoms of unknown etiology and nonspecific abdominal pain, especially of long duration. Such cases should prompt surgical interventions and workups as to not delay diagnosis and treatment of such a rare but possibly morbid disease process in adults that can lead to metastasis of malignancy and bowel ischemia if delayed.

\section{Acknowledgment}

Special thanks to the support of Western Michigan University Homer Stryker M.D. School of Medicine's medical librarians.

\section{Statement of Ethics}

The subject has given written informed consent to publish this report (including publication of images). This consent is available upon request. This case report was reviewed and deemed nonresearch by the WMU Homer Stryker M.D. School of Medicine Institutional Review Board. No protected health information or other uniquely identifying information is included in this manuscript.

\section{Conflict of Interest Statement}

The authors have no conflicts of interest to declare.

\section{Funding Sources}

This manuscript did not receive any funding.

\section{Author Contributions}

Z.Q., U.K., and S.S. all contributed to drafting the manuscript. Z.Q. formatted and submitted the manuscript. All authors reviewed the results and approved the final version of the manuscript.

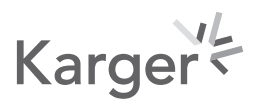




\section{References}

1 Demirkan A, Yağmurlu A, Kepenekci I, Sulaimanov M, Gecim E, Dindar H. Intussusception in adult and pediatric patients: two different entities. Surg Today. 2009 0ct;39(10):861-5.

2 Morais M, Pinho AC, Marques A, Lopes J, Duarte A, da Silva PC, et al. Mixed adenoneuroendocrine carcinoma causing colonic intussusception. Case Rep Surg. 2016;2016:7684364.

3 Honjo H, Mike M, Kusanagi H, Kano N. Adult intussusception: a retrospective review. World J Surg. 2015 Jan; 39(1):134-8.

4 Laham L, Bhattacharyya R, Guerrero M, Haghshenas J, Ingram M. Jejunal intussusception: a rare adult presentation of lymphoid hyperplasia. Case Rep Surg. 2019 Apr.

5 Gayer G, Zissin R, Apter S, Papa M, Hertz M. Pictorial review: adult intussusception - a CT diagnosis. Br J Radiol. 2002 Feb;75(890):185-90.

6 Takeuchi K, Tsuzuki Y, Ando T, Sekihara M, Hara T, Kori T, et al. The diagnosis and treatment of adult intussusception. J Clin Gastroenterol. 2003 Jan 1;36(1):18-21.

7 Marinis A, Yiallourou A, Samanides L, Dafnios N, Anastasopoulos G, Vassiliou I, et al. Intussusception of the bowel in adults: a review. World J Gastroenterol. 2009 Jan 28;15(4):407.

8 Mohamed M, Elghawy K, Scholten D, Wilson K, McCann M. Adult sigmoidorectal intussusception related to colonic lipoma: a rare case report with an atypical presentation. Int J Surg Case Rep. 2015 Jan 1;10:134-7.

9 Lvoff N, Breiman RS, Coakley FV, Lu Y, Warren RS. Distinguishing features of self-limiting adult small-bowel intussusception identified at CT. Radiology. 2003 Apr;227(1):68-72.

10 Brayton D, Norris WJ. Intussusception in adults. Am J Surg. 1954 Jul 1;88(1):32-43. 